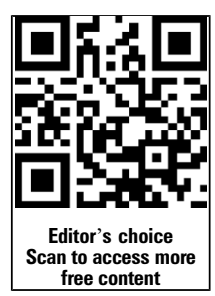
free content
frean to access $m$

For numbered affiliations see end of article.

\section{Correspondence to Professor Andrew J Lees, Reta Lila Weston Institute of Neurological Studies, UCL Institute of Neurology, 1 Wakefield street, London WC1N 1PJ, UK; andrew.lees@ucl.ac.uk}

SK and HLKY contributed equally to the manuscript.

Received 14 March 2015 Revised 28 May 2015 Accepted 17 June 2015 Published Online First 24 July 2015

\title{
Parkinson's disease without nigral degeneration: a pathological correlate of scans without evidence of dopaminergic deficit (SWEDD)?
}

\author{
Helen Ling, ${ }^{1,2}$ Seamus Kearney, ${ }^{1,3}$ Helen Lai Kuen Yip, ${ }^{4,5}$ Laura Silveira-Moriyama, 1,6 \\ Tamas Revesz, ${ }^{1,2}$ Janice L Holton, ${ }^{1,2}$ Catherine Strand ${ }^{2}$ Karen Davey, $^{2}$ \\ Kin Ying Mok, ${ }^{7,8}$ James M Polke, ${ }^{7}$ Andrew J Lees ${ }^{1,2}$
}

\begin{abstract}
Objective To describe 5 cases of Parkinson's disease lacking any detectable histopathology.

Background The diagnosis of Parkinson's disease is supported histologically by the findings of $\alpha$-synuclein immunopositive Lewy bodies and neurites and severe substantia nigra cell loss. Bradykinesia as defined by slowness of initiation of movement and a progressive reduction in speed and amplitude on finger tapping is a clinical correlate of pars compacta nigral degeneration. There are very few published cases of Parkinson's disease in which no pathological abnormality was found, and some of these cases were in hindsight thought to have probably been cases of indeterminate senile tremor or dystonic tremor.
\end{abstract}

Methods Retrospective case notes review of the Queen Square Brain Bank archival collection and detailed neuropathological analysis of the selected cases.

Results 5 cases considered to have Parkinson's disease by neurologists throughout the entirety of their illness that lacked any histopathological findings known to be associated with Parkinson's syndromes were identified out of a total number of 773 brains with a final clinical diagnosis of Parkinson's disease in the Queen Square Brain Bank. Retrospective case note analysis did not suggest dystonic tremor or indeterminate tremor in any of them. There was a reduction in tyrosine hydroxylase $(\mathrm{TH})$ density in the striatum in these cases when compared with healthy controls, but not in the substantia nigra.

Conclusions Striatal dopamine deficiency without nigral cell loss is the most likely explanation for the clinical findings; other possible explanations include slowness due to comorbidities misinterpreted as bradykinesia, a tardive syndrome related to undisclosed previous neuroleptic exposure, or 'soft age-related' parkinsonian signs. These cases emphasise the need to regularly review the diagnosis in cases of suspected Parkinson's disease and highlight the need for precision in the neurological examination particularly of elderly patients. These cases may represent a distinct entity of diagnostic exclusion and may be considered one explanation for the radiological phenomenon of SWEDD (scans without evidence of dopaminergic deficit).

\section{INTRODUCTION}

The diagnosis of Parkinson's disease is supported at autopsy by the finding of severe loss of neurons in the pars compacta of the substantia nigra associated with $\alpha$-synuclein immunopositive Lewy bodies and neurites. ${ }^{1}$ There are a few case reports of Parkinson's disease in which no pathological abnormality was found despite a detailed histological examination. ${ }^{2-6}$ We now report five patients who were considered to have Parkinson's disease throughout the entirety of their illness, ${ }^{7-9}$ but at postmortem lacked any histopathological findings associated with any recognised neurodegenerative or toxic cause of parkinsonism.

\section{PATIENTS AND METHODS}

Protocols used for brain donation in the Queen Square Brain Bank (QSBB) were approved by a London Multi-Centre Research Ethics Committee and written consent was obtained from all cases. Tissue is stored at the QSBB under a license from the Human Tissue Authority.

\section{Clinical data}

Between 1989 and the end of 2014, there were 773 brain donors diagnosed with Parkinson's disease received at the QSBB. Five cases were identified in which the detailed clinical documentation was highly suggestive of Parkinson's disease and in which the pathological diagnosis of Parkinson's disease, other neurodegenerative parkinsonian syndromes and vascular parkinsonism had been excluded at autopsy. All five selected cases had correspondence between neurologists and the general practitioner (GP), a full GP record and the patient's full prescription record obtained from the GP practice. Cases with drug-induced parkinsonism, whose clinical manifestations were considered to be directly related to medications such as dopamine receptor antagonists, were excluded from this study. Two of the selected cases (cases 2 and 3) had a history of short-lived prochlorperazine use for 'giddiness'. Two cases were preregistered brain donors and had received standardised prospective annual clinical assessments (cases 4 and 5), one attended a regional neurological centre (case 3 ) and three (cases 1,2 and 5) were followed up regularly throughout their illness in movement disorder clinics.

Complete clinical notes were obtained on all patients and a retrospective case notes review was undertaken by three QSBB-associated clinicians 
(HL, SK and HLKY) who formulated independent clinical summaries. Consensus opinion on each case was reached by two other clinicians (AJL and LS-M). None of the patients had dopamine transporter scans and no video recordings of the patients were available.

\section{Neuropathological methods}

Immediately after postmortem, the brains were divided in the mid-sagittal plane. One half, chosen randomly, was sliced and tissue blocks were frozen and stored, while the other half was immersed and fixed in 10\% neutral formalin for 3 weeks before neuropathological examination. Tissue blocks were taken using standard QSBB protocols. $8 \mu \mathrm{m}$ Thick histological sections were stained with the H\&E method. Immunohistochemistry with antibodies to $\alpha$-synuclein (frontal, parietal and temporal cortices, hippocampus, amygdala, midbrain, pons), A $\beta$ (frontal, parietal and temporal cortices, hippocampus, caudate, putamen, globus pallidus, midbrain, pons, cerebellum), phospho- $\tau$ (AT8; frontal, parietal and temporal cortices, hippocampus, amygdala, caudate, putamen, globus pallidus, subthalamic nuclei, midbrain, pons, cerebellum), p62 (frontal cortex, hippocampus, caudate, putamen, globus pallidus, cerebellum) and transactive response (TAR) DNA-binding protein 43 (TDP-43; hippocampus, amygdala) was carried out in selected brain regions using the standard avidin-biotin method. Immunohistochemistry with tyrosine hydroxylase antibody ( $\mathrm{TH}$, caudate, putamen, midbrain) was performed in five age-matched and disease duration-matched Parkinson's disease cases and five age-matched healthy controls.

Systematic histological analysis of neuronal loss in the substantia nigra and examination of the immunohistochemistry sections in search of inclusions known to be associated with clinical parkinsonism were performed by a neuropathologist (TR). Neuronal loss in the substantia nigra was determined using a four-tier semiquantitative grading $(0=$ absent, $1=$ mild, $2=$ moderate, 3 =severe). Additional pathologies including cerebrovascular disease and cerebral amyloid angiopathy and Alzheimer's disease pathological change were carefully assessed. $^{10}$

\section{Image analysis of TH immunoreactivity}

Using coded slides, quantitative assessment of TH immunoreactivity was performed in four subregions of the striatum (dorsal putamen, ventral putamen, dorsal caudate and ventral caudate) and four subregions of the substantia nigra pars compacta (dorsal lateral, ventral lateral, dorsal medial and ventral medial). The images of the striatum and substantia nigra pars compacta using $\times 20$ objective were captured by a high-resolution digital scanner and processed with image analysis software (Definiens TissueMap image analysis software V.3.0, Definiens AG, Germany). The threshold was adjusted to capture the twodimensional area of all $\mathrm{TH}$ immunoreactivity and the same threshold setting was used for all the subregions in all cases included in the analysis. 'Areal fraction', defined by a ratio of the TH immunoreactive pixels to the total number of pixels in the whole field, was computed by the image analysis software and was expressed as a percentage (areal fraction $\times 100 \%){ }^{11}$

\section{Statistical analysis}

The Mann-Whitney U test was used to compare the areal fraction percentage between cases in the present series and control groups. In this exploratory study, statistical significance was set at $\mathrm{p}<0.05$ and results were not adjusted for multiple comparisons. The $\chi^{2} /$ Fisher's exact test, the Student $t$ test or one-way analysis of variance (ANOVA) was used to compare semiquantitative grading or demographic data using the $\mathrm{p}$ value of 0.05. The SPSS V.22.0 program (IBM Corporation, New York, New York, USA) was used for statistical analysis.

\section{RESULTS}

All five patients had presenting clinical features and a disease course highly suggestive of Parkinson's disease. Four of them presented with a levodopa-responsive asymmetrical rest tremor. The mean age at onset was 70.8 years (range 55-83) and the mean disease duration was 11 years (range 4-18). None of the cases had a positive family history of movement disorder. Other clinical demographics are summarised in table 1. Only one case (case 5) had neuroimaging study; CT of the head was performed 2 years before death following a fall but no structural abnormalities were reported.

\section{Case reports}

Case 1

An 86-year-old man presented to his GP in 2006 with a 3-year history of gradually worsening right hand tremor and a 6-month history of poor balance and 'giddiness' on standing up. A pill rolling tremor of the right hand, bilateral cogwheel rigidity and shuffling gait were observed by his GP and a diagnosis of Parkinson's disease was made. He was subsequently referred to a specialist movement disorder clinic, and 1 year after his first symptoms, he was noted to have had a sustained marked improvement following treatment with levodopa/carbidopa $62.5 \mathrm{mg}$ four times daily. A festinant gait, reduced arm swing and bilateral, asymmetrical cogwheel rigidity were now noted. Ropinirole $1 \mathrm{mg}$ three times daily was added and a further symptomatic improvement in stiffness and mobility occurred, but he developed light-headedness due to orthostatic hypotension. At this stage, he was recruited to a Parkinson's disease trial. He remained under regular review with slow but progressive motor deterioration until he died at age 88 from a pulmonary embolism secondary to lung carcinoma, 5 years after his clinical diagnosis of Parkinson's disease.

\section{Case 2}

In 2001, a 78-year-old woman was diagnosed with Parkinson's disease by a consultant neurologist at a movement disorder clinic after presenting with a 10-month history of progressive worsening of general slowness and a tremor at rest. On examination, a marked bilateral rest tremor of the hands was observed in addition to a head tremor, bilateral bradykinesia, cogwheel rigidity, reduced blink rate and impaired postural reflexes. She had difficulty rising from a chair and her gait was of reduced stride length, with episodes of freezing. Levodopa/carbidopa $125 \mathrm{mg}$ three times daily was started but at review, 2 months later, her condition was thought to have deteriorated. She started to have occasional falls backwards. The medication records from the GP showed that she was prescribed prochlorperazine $5 \mathrm{mg}$ for a few weeks for vertigo during the period of reported rapid deterioration. The prochlorperazine prescription was not repeated and her levodopa dose was gradually increased to $750 \mathrm{mg}$ daily, and over the following 2 years some of the doctors who saw her considered her to have improved, while others commented that her parkinsonism had stabilised. She reported a subjective worsening of her symptoms at the end of each levodopa dose, but she did not experience clear benefit from individual doses, and on one occasion she inadvertently decreased her dose and did not notice any worsening. She died 4 years after the diagnosis of Parkinson's disease from bronchopneumonia at age 81 . 
Table 1 Clinical demographics of the five cases included in this case series

\begin{tabular}{|c|c|c|c|c|c|}
\hline & Case 1 & Case 2 & Case 3 & Case 4 & Case 5 \\
\hline Gender & Male & Female & Female & Male & Female \\
\hline Age at onset & 83 & 77 & 59 & 55 & 80 \\
\hline $\begin{array}{l}\text { Age at death } \\
\text { (disease duration) }\end{array}$ & $88(5)$ & $81(4)$ & $72(13)$ & $73(18)$ & $95(15)$ \\
\hline Initial presentation & Rest tremor (right hand) & Rest tremor (left hand), parkinsonian gait & Rest tremor (right hand) & Generalised slowing & Bilateral hand tremor \\
\hline $\begin{array}{l}\text { Other symptoms and } \\
\text { signs }\end{array}$ & $\begin{array}{l}\text { Asymmetric rest hand tremor, rigidity and } \\
\text { reduced arm swing, festinant gait, falls, } \\
\text { postural hypotension, abnormal glabellar } \\
\text { response }\end{array}$ & $\begin{array}{l}\text { Asymmetric rest hand tremor, head and } \\
\text { leg tremor, bradykinesia, general } \\
\text { slowness, rigidity, gait freezing, falls, } \\
\text { hypomimia }\end{array}$ & $\begin{array}{l}\text { Asymmetric rest hand tremor, rigidity, slow } \\
\text { handwriting, general slowness, rigidity, } \\
\text { postural instability (wheelchair use at age 69) }\end{array}$ & $\begin{array}{l}\text { Asymmetric bradykinesia and rigidity, } \\
\text { occasional rest hand tremor, } \\
\text { hypomimia, abnormal glabellar } \\
\text { response, hypomimia }\end{array}$ & $\begin{array}{l}\text { Head and leg tremor, rigidity, } \\
\text { postural instability and recurrent } \\
\text { falls (age 93), dysphagia (age } \\
\text { 94) }\end{array}$ \\
\hline Predominant tremor & Yes & Yes & Yes & No & Yes \\
\hline $\begin{array}{l}\text { Initial levodopa } \\
\text { response }\end{array}$ & Excellent (improved all motor symptoms) & Mild & Moderate & Good & Good \\
\hline $\begin{array}{l}\text { Sustained levodopa } \\
\text { response }\end{array}$ & Yes & Yes & Yes & Yes & Yes \\
\hline $\begin{array}{l}\text { Maximum levodopa } \\
\text { equivalent dose, } \\
\mathrm{mg} / \text { day }\end{array}$ & 320 & 1200 & 1250 & 1200 & 400 \\
\hline Motor fluctuation & No & Mild wearing off & $\begin{array}{l}\text { Generalised twitching movements at peak } \\
\text { dose, wearing off }\end{array}$ & No & $\begin{array}{l}\text { Mild choreiform movements of } \\
\text { head at peak dose, wearing off }\end{array}$ \\
\hline Visual hallucinations & No & No & No & No & No \\
\hline Memory impairment & No & No & No & $\begin{array}{l}\text { Mild cognitive impairment (MMSE } \\
\text { scores: 23-30/30) }\end{array}$ & No \\
\hline Disease progression & $\begin{array}{l}\text { Slow progression only before levodopa } \\
\text { with subsequent plateau }\end{array}$ & $\begin{array}{l}\text { Initial rapid progression with subsequent } \\
\text { plateau }\end{array}$ & Gradual deterioration & No progression & Gradual deterioration \\
\hline Comorbidities & Ischaemic heart disease & Depression, COPD & $\begin{array}{l}\text { Anxiety, depression, hemifacial spasm, } \\
\text { osteoarthritis, osteoporosis, cervical } \\
\text { spondylosis, chronic back pain, Sjögren's } \\
\text { syndrome, asthma }\end{array}$ & $\begin{array}{l}\text { Anxiety, migraine, ischaemic heart } \\
\text { disease }\end{array}$ & $\begin{array}{l}\text { Ischaemic heart disease, } \\
\text { pulmonary tuberculosis }\end{array}$ \\
\hline $\begin{array}{l}\text { Features against } \\
\text { Parkinson's disease }\end{array}$ & No record of bradykinesia & $\begin{array}{l}\text { Head tremor, mild levodopa response, } \\
\text { transient use of prochlorperazine } \\
\text { (coincided with worsening of } \\
\text { parkinsonism) }\end{array}$ & $\begin{array}{l}\text { No record of bradykinesia, intermittent use of } \\
\text { prochlorperazine (stopped when first } \\
\text { diagnosed with Parkinson's disease) }\end{array}$ & $\begin{array}{l}\text { Mild motor symptoms, lack of } \\
\text { progression }\end{array}$ & $\begin{array}{l}\text { Head tremor, lack of asymmetry } \\
\text { or record of bradykinesia }\end{array}$ \\
\hline
\end{tabular}


Figure 1 An example of the handwriting of case 2 . A letter written by the patient at age 68 to her general practitioner 8 years after the onset of her initial symptoms of asymmetric rest tremor of the hands. There is no evidence of micrographia but the patient reported slowness.

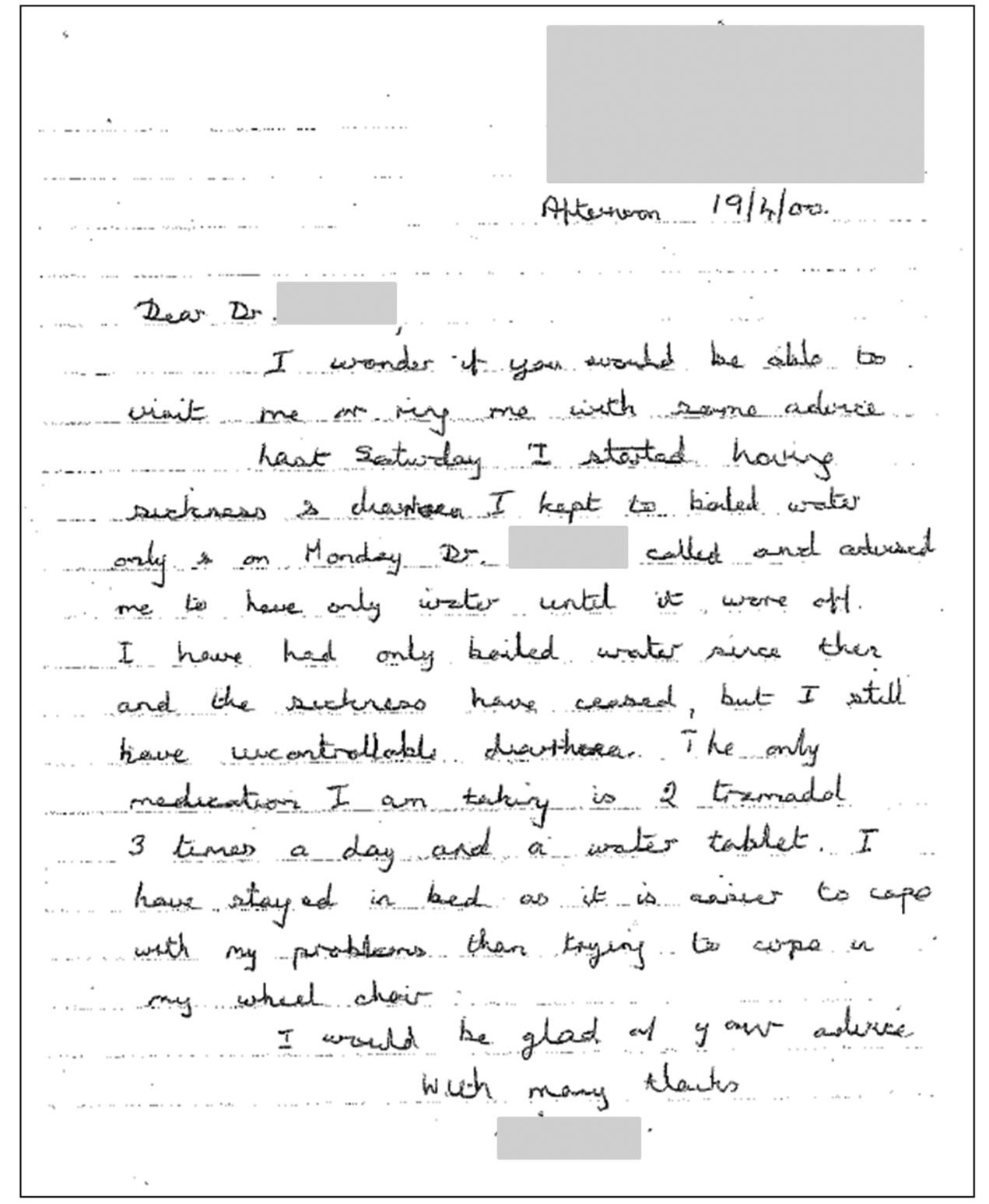

\section{Case 3}

In 1992, a 59-year-old woman with a history of several nonspecific symptoms was diagnosed with 'parkinsonism' by her GP after finally presenting with limb stiffness and a right-sided tremor. She was taking prochlorperazine, which was withdrawn, and she was subsequently seen by a consultant neurologist who documented 'parkinsonism with a right-sided emphasis' and started on levodopa/carbidopa $62.5 \mathrm{mg}$ twice daily. She reported slowing of her handwriting, but a letter written by her to the GP did not reveal micrographia (figure 1). After several years of follow-up she was considered to have 'benign tremulous parkinsonism'. Her levodopa dose was gradually increased, reaching a peak of $1250 \mathrm{mg}$ daily, when she reported unilateral involuntary facial movements that were thought to be levodopa-induced dyskinesias, but were later diagnosed as hemifacial spasm. A dispersible formulation of levodopa/benserazide was prescribed to treat nocturnal rigidity. Progressive deterioration in her mobility due to a combination of Parkinson's disease and lumbar degenerative disease led her to be wheelchair dependent 8 years after her diagnosis of Parkinson's disease. In the last year of her life, there were reports in her notes of involuntary wild 'twitching' movements following the intake of levodopa. She continued to be treated for Parkinson's disease for 12 years until she died from bronchopneumonia and heart failure at age 71 .

\section{Case 4}

A 55-year-old was diagnosed with Parkinson's disease in 1976 by his GP after presenting with limb stiffness. Examination 2 years after the initial diagnosis revealed a 'mask-like face', a positive glabellar tap response and rigidity in the limbs with a right-sided predominance. He was started on levodopa/carbidopa $110 \mathrm{mg}$ twice daily 2 years after diagnosis. At his first assessment by a consultant neurologist, the patient reported his initial response to levodopa to be excellent. Seven consecutive annual QSBB prospective assessments all reported mild to moderate, usually symmetrical, bradykinesia and rigidity with no deterioration in his Hoehn and Yahr stage from the baseline level of 2. His levodopa dose was gradually titrated up to $750 \mathrm{mg}$ per day, but he did not experience motor fluctuations or dyskinesias. He died from bronchopneumonia complicating myeloma after 18 years of disease. 
Case 5

A woman was diagnosed with Parkinson's disease by her GP in 1987 at the age of 84 after presenting with a 4-year history of progressive bilateral rest tremor of the hands. An initial QSBB assessment form filled in by an affiliated neurologist in 1993 reported a Hoehn and Yahr stage of 2. Examination by a neurologist revealed mild bilateral limb rigidity and severe tremor in the hands and legs. She was started on levodopa/carbidopa $125 \mathrm{mg}$, which was gradually titrated up to four times daily. She reported a good response to levodopa therapy. Three years later, she noted mild choreiform movements of her head and neck on reaching the maximum levodopa dose, and a transient worsening of her motor symptoms coincided with the end of each dose of levodopa. Between 1990 and 1997, she was regularly followed up in a Parkinson's disease clinic, and progressive deterioration in the tremor of her head and hands, mobility and gait freezing was documented. At the age of 93, she developed marked postural instability, freezing of gait and falls. She could only walk a few steps using a walking frame. She reported drooling of saliva and difficulty in swallowing. She died after 15 years of disease and the cause of death was recorded as 'endstage Parkinson's disease'.

\section{Pathological findings}

None of the cases was deemed to have a pathological explanation for parkinsonism. No cases met the criteria for the pathological diagnosis of Parkinson's disease, vascular parkinsonism or other neurodegenerative parkinsonian syndromes. Staining for $\alpha$-synuclein was negative in the medulla and substantia nigra in all cases, confirming the absence of Lewy body pathology. Macroscopic examination was unremarkable with normal pigmentation of the substantia nigra and absence of cortical atrophy or ventricular dilation. Mild nigral cell loss compatible with ageing was found in three cases (1, 3 and 4) with evidence of negligible free pigment (1 and 3 ). The substantia nigra in the remaining two cases was normal (2 and 5; figure 2 ). The locus

A case example of

Parkinson's disease
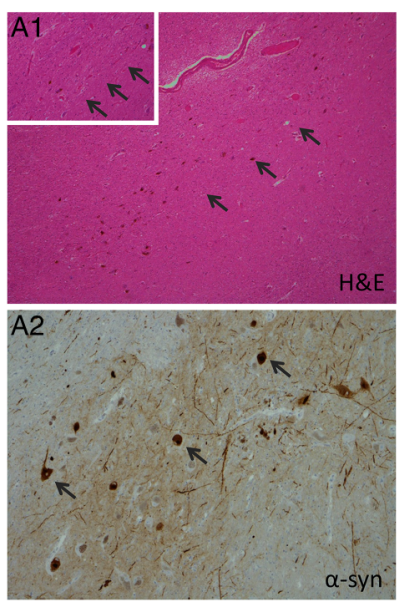

Figure 2 Substantia nigra of a case example of Parkinson's disease and case 2 . In the Parkinson's disease case, severe degree of cell loss of pigmented neurons and gliosis (A-1, $\times 4$ objective) and Lewy bodies and Lewy neurites containing $\alpha$-synuclein ( $\alpha$-syn, A-2, $\times 10$ objective) in the substantia nigra are evident. In case 2 , the substantia nigra is well preserved without evidence of neuronal loss or gliosis (B-1, $\times 4$ objective) and no Lewy bodies or Lewy neurites are identified using $\alpha$-syn immunohistochemistry (B-2, $\times 4$ objective). coeruleus was normal in four cases (cases 1, 2, 4 and 5) but was unavailable for examination in case 3 . The subthalamic nucleus, corpus striatum and thalamus were normal in all five cases with no cell loss or pathological inclusions.

No cases exhibited extranigral pathology, which was deemed to be sufficient to cause clinical parkinsonism. In two cases (cases 1 and 3), there was mild mineralisation of blood vessels in the globus pallidus. Case 1 also had mild hyaline arteriolar thickening in the deep white matter and mild cerebral amyloid angiopathy, with the extent of vascular change being slightly more than expected for the patient's age. Mild perivascular accentuation associated with a mild degree of gliosis in the striatum was observed in four cases (1-4). These findings are common in older individuals and were not of the degree observed previously in vascular parkinsonism cases; ${ }^{12}{ }^{13}$ they were also judged to be not severe enough to account for parkinsonism. Mild age-related Alzheimer's type $\tau$ pathology was identified in all cases at Braak and Braak stage II or below. ${ }^{10}$ Immunohistochemistry with TDP-43 was negative in all cases.

Neuropathological examination including immunohistochemistry excluded any structural causes and confirmed the absence of any distinctive histological hallmarks for any of the neurodegenerative entities listed in box 1. Genetic analysis performed in all five cases using DNA obtained from frozen brain tissue excluded mutation of the Huntington's disease gene (repeat size 18-26).

\section{Image analysis of TH immunoreactivity}

TH synthesises dopamine from tyrosine and TH immunohistochemistry is used as a marker of catecholaminergic fibres including dopaminergic neurons.

Quantitative assessment of the density of TH immunoreactivity was performed in these five cases (mean age of death: 81.8 years, mean disease duration: 11 years), five age-matched and disease duration-matched Parkinson's disease controls (mean age of death: 79.8 years, $\mathrm{p}=0.32$; mean disease duration: 14.2 years, $\mathrm{p}=0.76$ ) and 5 age-matched healthy controls (mean age of death: 77.0 years, $p=0.16) .{ }^{14}$

In all subregions of the substantia nigra pars compacta, there was reduction in the TH density in Parkinson's disease when compared with the present cohort (dorsolateral: $p=0.009$,

Box 1 Potential explanations for Parkinson's disease mimics without nigral atrophy and Lewy body pathology.

Neurodegenerative causes:

- Huntington's disease

- Fragile X tremor ataxia syndrome (FXTAS)

- Chronic traumatic encephalopathy

- C9orf72 expansion

- Spinocerebellar ataxia (SCA)

Non-neurodegenerative causes:

- Mild extrapyramidal signs of the elderly

- Indeterminate or senile tremor

- Dystonic tremor

- Tardive parkinsonism

- Vascular parkinsonism

- Psychomotor retardation due to depression

- Rheumatological or orthopaedic conditions (eg, arthritis, spondylosis) 
Figure 3 Substantia nigra of a healthy control, case 1 and a Parkinson's disease (PD) control $(\times 4$ objective). Severe neuronal loss, gliosis and markedly reduced tyrosine hydroxylase $(\mathrm{TH})$ density can be observed in the PD case. TH density in the substantia nigra is preserved in case 1 and healthy control.

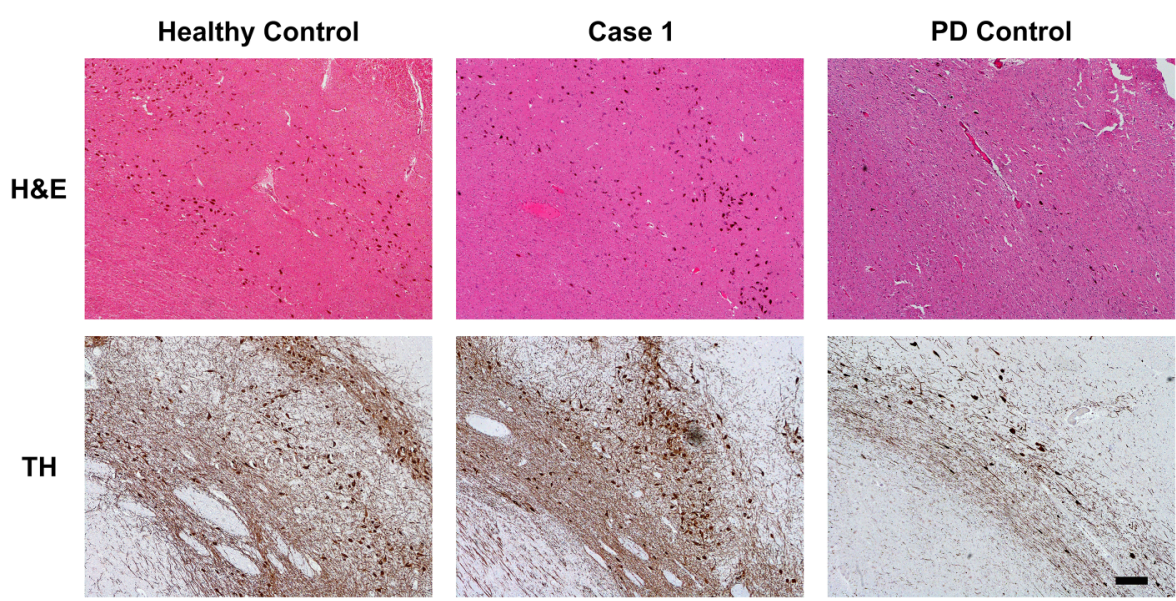

ventrolateral: $p=0.009$, dorsomedial: $p=0.028$, ventromedial: $p=0.009$ ) and the healthy controls (dorsolateral: $p=0.014$, ventrolateral: $p=0.014$, dorsomedial: $p=0.014$, ventromedial: $\mathrm{p}=0.027$ ). The TH density was the same between the present cohort and healthy controls (dorsolateral: $\mathrm{p}=0.806$, ventrolateral: $p=0.462$, dorsomedial: $p=0.624$, ventromedial: $p=0.806$; figure 3).

In the striatum, the $\mathrm{TH}$ density in the dorsal putamen $(p=0.034)$, ventral putamen (borderline significance; $p=0.077)$ and ventral caudate $(p=0.034)$ of the present cohort was less than that of the healthy controls, but not in the dorsal caudate $(p=0.289)$. The TH density in the present cohort was numerically greater than Parkinson's disease controls, but it did not reach statistical significance (dorsal putamen: $p=0.149$, ventral putamen: $p=0.149$, dorsal caudate: $p=0.248$, ventral caudate: $\mathrm{p}=0.386$; figures 4 and 5).

\section{DISCUSSION}

It came as a surprise that with the use of dopamine transporter single-photon emission CT (SPECT) scans, up to $15 \%$ of patients in trials for early Parkinson's disease had no evidence of presynaptic nigrostriatal dopamine denervation. ${ }^{15-17}$ The radiological acronym SWEDD (scans without evidence of dopaminergic deficit) was used to describe these patients, and there is ongoing debate regarding their true diagnosis. Repeat dopamine transporter scans often remain normal with only $8-13 \%$ converted to have an abnormal scan up to 5 years later. ${ }^{18-20}$ Almost half of the patients with SWEDD (40/90) in the PRECEPT Parkinson's disease clinical trial $(\mathrm{N}=799)$ had their diagnosis changed to a condition not associated with dopamine denervation at 22-month follow-up by study investigators blinded to the dopaminergic scan status with the most common revised diagnosis being tremor, followed by 'no neurological diagnosis' and vascular parkinsonism, ${ }^{19}$ highlighting the diagnostic difficulties in the early disease stage, and even in
Parkinson's disease patients with SPECT-confirmed dopaminergic deficits $(\mathrm{N}=707), 5$ cases had been re-evaluated as having a tremor syndrome not associated with dopamine denervation and 13 had their diagnosis revised to vascular parkinsonism. ${ }^{19}$ Patients with SWEDD generally had a milder motor disability as measured by the Unified Parkinson's Disease Rating Scale (UPDRS), ${ }^{19}$ their clinical symptoms tended to be stable with no deterioration after withdrawal of levodopa therapy ${ }^{19}$ and they present with less non-motor features, ${ }^{21} 22$ all of which might be useful clinical pointers. Most neurologists now consider that these patients do not have Parkinson's disease. ${ }^{18} 19$ Some of the tremulous patients with SWEDD can present with dystonic features $^{23}$ and abnormal cortical plasticity, ${ }^{21}$ suggesting that dystonic tremor could be the underlying diagnosis. Patients with dystonic and indeterminate senile tremor may have reduced arm swing, small stride length, asymmetric jerky rest and postural tremor, hypomimia, increased limb tone and slow repetitive finger movements, and it has been claimed that 3\% of dystonic tremor are misdiagnosed with Parkinson's disease. ${ }^{23-25}$ Nevertheless, many patients with SWEDD do not fall into this phenotype and pathological findings in SWEDD have not been published.

Slowness of initiation of movement or reduced amplitude of movements without motor decrement may be seen in dystonia or pyramidal slowness, ${ }^{24} 26$ but bradykinesia with motor decrement and fatiguing has been considered a clinical correlate of nigral deficiency. The QSBB diagnostic criteria stipulate that the presence of bradykinesia is obligatory for the diagnosis of parkinsonism. ${ }^{7}$ On re-examination, some patients with SWEDD from the clinical series exhibited slowness or reduction of amplitude but no true motor decrement, ${ }^{21}{ }^{23}$ highlighting the importance of repetitive finger tapping tests performed for $15-30 \mathrm{~s}$ in the clinical differentiation between patients with Parkinson's disease and SWEDD. ${ }^{27}$ Nevertheless, a recent blinded video study showed that even experienced movement
Figure 4 Tyrosine hydroxylase (TH) immunohistochemistry in the dorsal putamen ( $\times 4$ objective) of a healthy control, case 1 and a Parkinson's disease (PD) control. TH density is markedly reduced in the PD control and mildly reduced in case 1 when compared with healthy control.
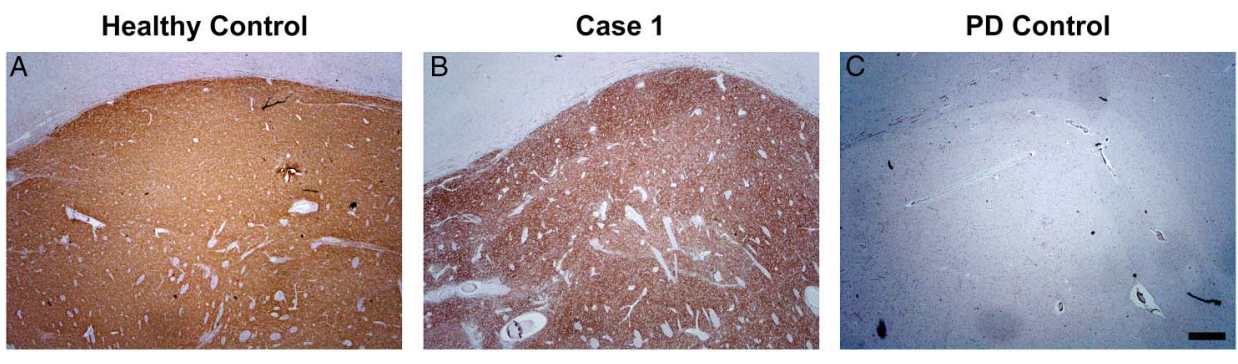
Figure 5 Tyrosine hydroxylase (TH) immunohistochemistry in four subregions of the striatum $(\times 20$ objective) of a healthy control, case 1 and a Parkinson's disease (PD) control. When compared with healthy controls, TH density is markedly reduced in the striatum in PD controls and, to a lesser extent, in the cases in this series.
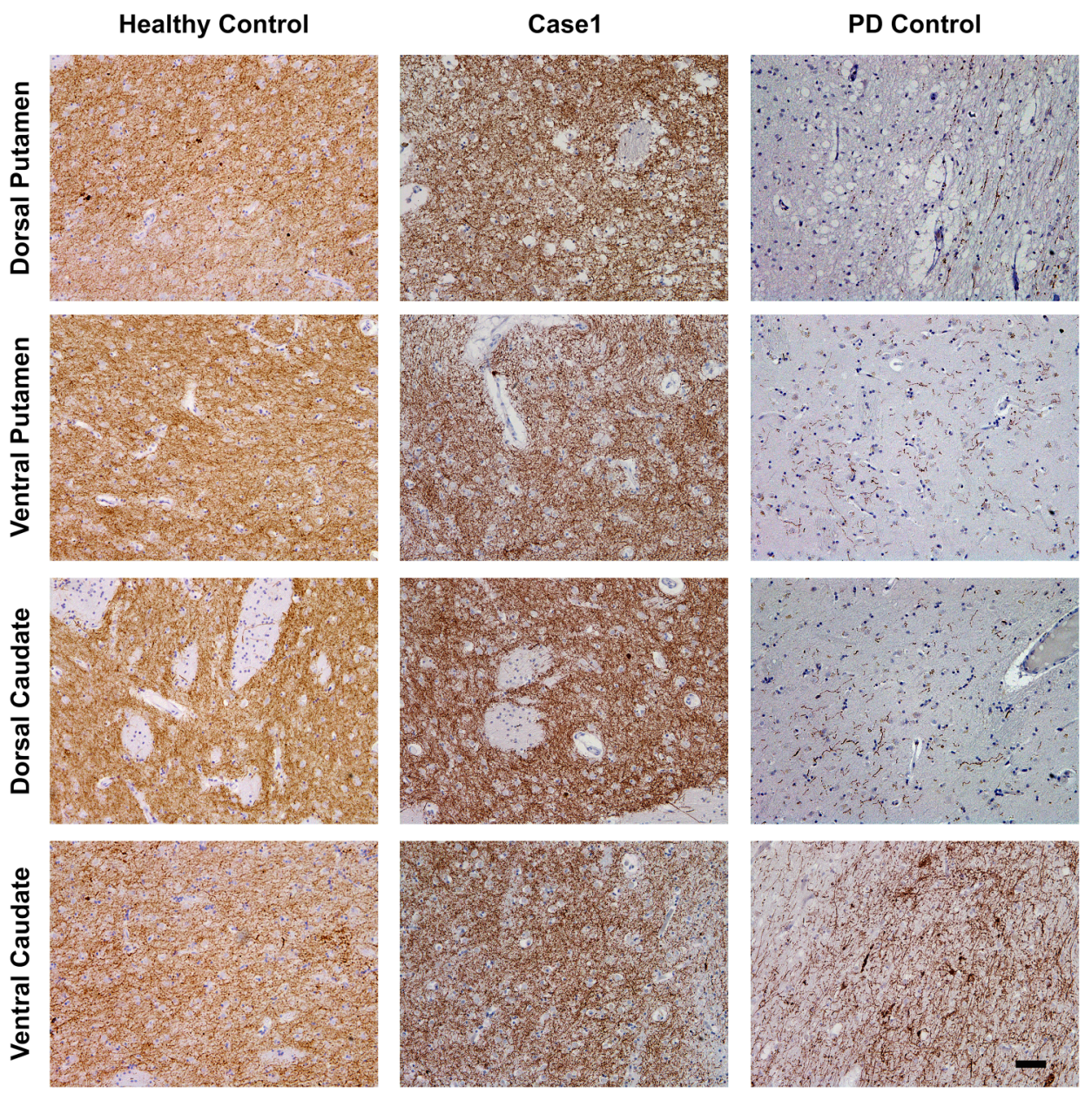

disorder specialists may have difficulty distinguishing slowness in SWEDD from bradykinesia in Parkinson's disease. ${ }^{28}$ Our findings support this area of clinical uncertainty in that three of our patients were considered to have either bradykinesia (cases 2 and 4) or general slowness (case 3). We cannot exclude the possibility that slowness due to ageing or comorbidities (depression, arthritis, spondylosis) was misinterpreted as criteriadefined bradykinesia in some of these cases.

One could argue that the slow rate of deterioration should have cast doubt on the clinical diagnosis of Parkinson's disease. Case 4 had Parkinson's disease for 18 years with little progression. Cases 3 and 5 had a gradual deterioration over 13 and 15 years. Case 2 initially had a rapidly progressive course, during which she was also receiving prochlorperazine, followed by a "stabilisation of symptoms" after prochlorperazine withdrawal. In contrast, case 1 exhibited a very typical course for Parkinson's disease, with a rest tremor at onset that progressed to a shuffling gait in the pretreatment phase, but no further progression was reported after starting dopaminergic medication. Although Parkinson's disease is always progressive, heterogeneity exists in the rate of deterioration with some patients following a relatively mild course, remaining in stage 1 or 2 Hoehn and Yahr $^{29}$ for up to 10 years. Clinically, four of five of these cases had a tremor predominant clinical picture and can mimic benign tremulous parkinsonism, a pathologically proven subtype of Parkinson's disease which is associated with a relatively slow rate of clinical progression at least in the early or mid-disease stage. ${ }^{30}$ Our cases raise the possibility that a small proportion of those slowly progressive cases might in fact not have underlying nigral degeneration.
All the patients had some benefit from levodopa; case 1 had adequate documentation of sustained levodopa responsiveness, while cases 2, 3 and 5 exhibited wearing off effects described in the notes. Occasional episodes of generalised twitching movements were reported at peak dose in case 3 in the last year of life and mild choreiform head movements were noted at peak dose of levodopa therapy in case 5. Marked initial and sustained levodopa responsiveness are features supportive of the diagnosis of Parkinson's disease, with $75 \%$ of pathologically confirmed Parkinson's disease exhibiting a good or excellent initial levodopa response. ${ }^{7}$ The presence of severe levodopa-induced chorea has a high positive predictive value for the diagnosis of Parkinson's disease and is included as a supportive feature in Parkinson's disease diagnostic criteria. ${ }^{731} 32$ However, in pathologically proven Parkinson's disease, a poor or moderate levodopa response was noted in $17 \%$ of cases, and no dyskinesias or motor fluctuations were reported in 34\%. ${ }^{7}$ Furthermore, dyskinesias are less common in poorly levodopa-responsive patients or in the absence of motor fluctuations, with one recent clinicopathological study reporting dyskinesia in only $14 \%$ of nonfluctuators. ${ }^{33}$ The levodopa responses reported in our cases are clearly comparable to those seen in pathologically confirmed Parkinson's disease, highlighting further potential for diagnostic error.

Although not the primary emphasis of this paper, these cases provide a unique opportunity to observe clinical and pathological effects of long-term levodopa on healthy human substantia nigra. All patients received levodopa for at least 21 months (up to 216 months) in doses ranging from 200 to $1250 \mathrm{mg}$ 
daily. Although three patients reported side effects, the lack of pathological change in our cases provides further reassurance that levodopa is not toxic to human substantia nigra. ${ }^{4}$

The severe loss of dopamine-containing neurons from the pars compacta of the substantia nigra is the pathological hallmark of Parkinson's disease and the prerequisite for the development of motor symptoms. This loss of dopaminergic neurons is accompanied by Lewy bodies of which the main component is $\alpha$-synuclein. ${ }^{1}$ Unlike neurofibrillary tangles in tauopathies, a constant proportion of nigral neurons of 3-4\% contain Lewy bodies irrespective of the disease duration, supporting the notion that Lewy bodies are eliminated when the neurons that bear them die and that they are at the same time being constantly produced. ${ }^{34}$ On the basis of current clinicopathological concepts of disease, the patients in the present series cannot be said to have had Parkinson's disease. In most of the previously reported cases of Parkinson's disease without pathological explanation, the phenotype was predominantly tremulous, there was absent or debatable bradykinesia and rigidity ${ }^{3-5}$ and there was no significant response to levodopa with successful levodopa withdrawal in one case. ${ }^{5}$ Furthermore, in retrospect, there were sufficient features to support a revised clinical diagnosis of essential tremor, atypical tremor or dystonic tremor retrospectively. ${ }^{2}{ }^{5}$ In contrast, the cases in this series had cardinal motor features of Parkinson's disease and had been followed by neurologists for prolonged periods. The clinical presentations in cases 1,3 and 4 would be incompatible with essential tremor, atypical tremor or dystonic tremor. Although a head tremor was observed in cases 2 and 5, the impaired postural response and gait freezing would be unusual for indeterminate or senile tremor, and head tremor has been reported in Parkinson's disease. $^{35}$

TH density in our cases is reduced in the striatum but not in the substantia nigra. In the absence of neuronal loss in the substantia nigra and striatum as shown by semiquantitative assessment, the finding of reduced striatal TH immunohistochemistry suggests a biochemical deficiency of dopamine. In doparesponsive dystonia, there is selective nigrostriatal dopamine deficiency caused by genetic defects in the dopamine synthetic pathway with marked reduction of pigmented neurons but with absence of nigral degeneration and is considered a biochemical disorder in which symptoms can be reversed by replacement of the depleted neurochemicals. ${ }^{36}$ The reduction in striatal TH density in our cases could only be identified by sensitive stereological image analysis rather than the standard histopathological method, but we cannot exclude the possibility of mild regression of dopamine-containing terminals in these cases. Our findings, however, do not support the loss of the entire nigrostriatal dopaminergic neurons as in Parkinson's disease. Striatal dopamine deficiency is the most likely explanation for the clinical findings of parkinsonism and positive levodopa response in these cases. Its underlying cause, however, will warrant further study, which is beyond the scope of this series. Other credible arguments can be proposed as to why these patients had parkinsonism and in some cases responded to levodopa. Undisclosed continued neuroleptic use or a tardive phenomenon (cases 2 and 3) following neuroleptic exposure in two of the cases could also be speculated. ${ }^{37}$ All the patients were elderly and it may be that they had soft extrapyramidal signs related to ageing that were confused with Parkinson's disease. ${ }^{38} 39$

Dopamine transporter locates in the presynaptic membrane on the terminals of dopaminergic projections from the substantia nigra to the striatum, and it provides a marker for dopamine terminal innervation. ${ }^{40}$ Dopamine transporter SPECT is normal in dopa-responsive dystonia. ${ }^{4142}$ We speculate that the result of the scan in our cases would also have been reported as normal on visual assessment, but semiquantitative assessment might have revealed a subtle reduction in tracer uptake in the striatum. We conclude that these five cases probably represent a subgroup of SWEDD cases, and it may be reasonable to assume that SWEDD is an entity of diagnostic exclusion with several distinct causes. ${ }^{19}$ Our cases serve to highlight the need for ongoing clinicopathological research and highlight the need to review the clinical diagnosis, including the judicious use of dopamine transporter scans, even in patients with longstanding disease if atypical features exist.

\section{Author affiliations}

${ }^{1}$ Reta Lila Weston Institute of Neurological Studies, UCL Institute of Neurology, London, UK

${ }^{2}$ Queen Square Brain Bank for Neurological Disorders, UCL Institute of Neurology, London, UK

${ }^{3}$ Belfast Health and Social Care Trust, Belfast, Ireland

${ }^{4}$ Department of Rehabilitation and Extended Care, Wong Tai Sin Hospital TWGHS, Hong Kong, Hong Kong

${ }^{5}$ Neuro-medical Unit, Department of Medicine and Geriatrics, Kwong Wah Hospital TWGHs, Hong Kong, Hong Kong

${ }^{6}$ Postgraduate Program in Medicine, Universidade Nove de Julho, UNINOVE, São Paulo, Brazil

${ }^{7}$ Department of Molecular Neuroscience, UCL Institute of Neurology, London, UK ${ }^{8}$ Division of Life Science, Hong Kong University of Science and Technology, Hong Kong, Hong Kong

Contributors HL contributed to the drafting of the manuscript for content, and to the collection, analysis and interpretation of data. SK and HL-KY contributed to the drafting of the manuscript for content, and to the analysis and interpretation of data. LS-M and AJL contributed to the revision of the manuscript for content, and to the study concept and design, and to the analysis and interpretation of data. TR, $\mathrm{JLH}$ and K-YM contributed to the revision of the manuscript for content, and to the analysis and interpretation of data. CS and KD contributed to the collection and analysis of data. JMP contributed to the analysis and interpretation of data.

Competing interests $\mathrm{HL}$ has received grants from CBD Solutions, PSP (Europe) Association and Reta Lila Weston Fellowship. SK has received travel grants from Teva-Lundbeck and Britannia pharmaceuticals. He has received honoraria from UCB and Abbvie pharmaceuticals in the past 2 years. LS-M declares employment from Universidade Nove de Julho (Uninove). She has received grants from the Reta Lila Howard Foundation, FAPESP, FAEPEX-UNICAMP, Parkinson's UK and CNPq-CAPES, and travel grants from Genus, Ipsen and Abbott. She has received no royalties or honoraria from any pharmaceutical company for the past 2 years. TR is a consultant for Merck Serono pharmaceuticals and has received an honorarium from Merck Serono pharmaceuticals and grants from Alzheimer's Research UK, Multiple System Atrophy Trust and Parkinson's UK. JLH has received grants from Multiple System Atrophy trust, Alzheimer's Research UK and Parkinson's UK. KYM has received grants from CBD Solutions. AJL serves on the advisory board for Novartis, Teva, Meda, Boehringer Ingelheim, GSK, Ipsen, Lundbeck, Allergan, Orion, BIAL, Noscira and Roche pharmaceuticals and has received honoraria from Novartis, Teva, Meda, Boehringer Ingelheim, GSK, Ipsen, Lundbeck, Allergan, Orion, BIAL, Noscira and Roche pharmaceuticals and grants from the PSP Association, Weston Trust- the Reta Lila Howard Foundation.

Patient consent Obtained.

Ethics approval London Multi-Centre Research Ethics Committee.

Provenance and peer review Not commissioned; externally peer reviewed.

\section{REFERENCES}

1 Ince PG, Clark B, Holton JL, et al. Disorders of movement and system degenerations. In: Love S, Louis DN, Ellison DW, eds. Greenfield's neuropathology. 8th edn. London: Arnold, 2008:889-1030.

2 Quinn N. The "round the houses" sign in progressive supranuclear palsy. Ann Neurol 1996;40:951

3 Rajput AH. Levodopa prolongs life expectancy and is non-toxic to substantia nigra. Parkinsonism Relat Disord 2001;8:95-100.

4 Rajput $\mathrm{AH}$, Fenton $\mathrm{M}$, Birdi $\mathrm{S}$, et al. Is levodopa toxic to human substantia nigra? Mov Disord 1997;12:634-8.

5 Rajput AH, Robinson CA. Benign tremulous parkinsonism: a clinicopathological study. Mov Disord 2008;23:311-12.

6 Velickovic M, Lesser G, Purohit D, et al. Parkinson's disease without expected neuropathologic abnormality. J Am Med Dir Assoc 2004;5:407-9. 
7 Hughes AJ, Daniel SE, Kilford L, et al. Accuracy of clinical diagnosis of idiopathic Parkinson's disease: a clinico-pathological study of 100 cases. J Neurol Neurosurg Psychiatry 1992;55:181-4.

8 Hughes AJ, Daniel SE, Lees AJ. Improved accuracy of clinical diagnosis of Lewy body Parkinson's disease. Neurology 2001;57:1497-9.

9 Rajput AH, Rozdilsky B, Rajput A. Accuracy of clinical diagnosis in parkinsonism-a prospective study. Can J Neurol Sci 1991;18:275-8.

10 Braak H, Alafuzoff I, Arzberger T, et al. Staging of Alzheimer disease-associated neurofibrillary pathology using paraffin sections and immunocytochemistry. Acta Neuropathol 2006;112:389-404.

11 Gundersen HJ, Bendtsen TF, Korbo L, et al. Some new, simple and efficient stereological methods and their use in pathological research and diagnosis. APMIS 1988;96:379-94.

12 Glass PG, Lees AJ, Bacellar A, et al. The clinical features of pathologically confirmed vascular parkinsonism. J Neurol Neurosurg Psychiatry 2012;83:1027-9.

13 Zijlmans JC, Daniel SE, Hughes AJ, et al. Clinicopathological investigation of vascular parkinsonism, including clinical criteria for diagnosis. Mov Disord 2004; 19:630-40.

14 Kordower JH, Olanow CW, Dodiya HB, et al. Disease duration and the integrity of the nigrostriatal system in Parkinson's disease. Brain 2013;136(Pt 8):2419-31.

15 Fahn S, Oakes D, Shoulson I, et al. Levodopa and the progression of Parkinson's disease. N Engl J Med 2004;351:2498-508.

16 Markek K, Seibyl J, Parkinson Study Group. $\beta$-CIT scans without evidence of dopaminergic deficit (SWEDD) in the ELLDOPA-CIT and CALM-CIT Study: long-term imaging assessment. Neurology 2003;60(suppl 1):A298.

17 Whone AL, Watts RL, Stoessl AJ, et al. Slower progression of Parkinson's disease with ropinirole versus levodopa: The REAL-PET study. Ann Neurol 2003;54:93-101.

18 Batla A, Erro R, Stamelou M, et al. Patients with scans without evidence of dopaminergic deficit: a long-term follow-up study. Mov Disord 2014;29:1820-5.

19 Marek K, Seibyl J, Eberly S, et al. Longitudinal follow-up of SWEDD subjects in the PRECEPT Study. Neurology 2014;82:1791-7.

20 Marshall VL, Patterson J, Hadley DM, et al. Two-year follow-up in 150 consecutive cases with normal dopamine transporter imaging. Nud Med Commun 2006;27:933-7.

21 Schwingenschuh P, Ruge D, Edwards MJ, et al. Distinguishing SWEDDs patients with asymmetric resting tremor from Parkinson's disease: a clinical and electrophysiological study. Mov Disord 2010;25:560-9.

22 Silveira-Moriyama L, Mathias C, Mason L, et al. Hyposmia in pure autonomic failure. Neurology 2009;72:1677-81.

23 Schneider SA, Edwards MJ, Mir P, et al. Patients with adult-onset dystonic tremor resembling parkinsonian tremor have scans without evidence of dopaminergic deficit (SWEDDs). Mov Disord 2007;22:2210-15.

24 Bain PG. Dystonic tremor presenting as parkinsonism: long-term follow-up of SWEDDs. Neurology 2009;72:1443-5.
25 Mian OS, Schneider SA, Schwingenschuh P, et al. Gait in SWEDDs patients: comparison with Parkinson's disease patients and healthy controls. Mov Disord 2011;26:1266-73.

26 Norlinah IM, Bhatia KP, Ostergaard K, et al. Primary lateral sclerosis mimicking atypical parkinsonism. Mov Disord 2007;22:2057-62.

27 Ling $\mathrm{H}$, Massey LA, Lees AJ, et al. Hypokinesia without decrement distinguishes progressive supranuclear palsy from Parkinson's disease. Brain 2012;135(Pt 4):1141-53.

28 Bajaj NP, Gontu V, Birchall J, et al. Accuracy of clinical diagnosis in tremulous parkinsonian patients: a blinded video study. J Neurol Neurosurg Psychiatry 2010;81:1223-8.

29 Hoehn MM, Yahr MD. Parkinsonism: onset, progression and mortality. Neurology 1967:17:427-42

30 Shahim $\mathrm{P}$, Tegner $\mathrm{Y}$, Wilson $\mathrm{DH}$, et al. Blood biomarkers for brain injury in concussed professional ice hockey players. JAMA Neurol 2014;71:684-92.

31 Hughes AJ, Frankel JP, Kempster PA, et al. Motor response to levodopa in patients with parkinsonian motor fluctuations: a follow-up study over three years. J Neurol Neurosurg Psychiatry 1994;57:430-4.

32 McColl CD, Reardon KA, Shiff M, et al. Motor response to levodopa and the evolution of motor fluctuations in the first decade of treatment of Parkinson's disease. Mov Disord 2002;17:1227-34.

33 Kempster PA, Williams DR, Selikhova $M$, et al. Patterns of levodopa response in Parkinson's disease: a clinico-pathological study. Brain 2007;130(Pt 8):2123-8.

34 Greffard S, Verny M, Bonnet AM, et al. A stable proportion of Lewy body bearing neurons in the substantia nigra suggests a model in which the Lewy body causes neuronal death. Neurobiol Aging 2010;31:99-103.

35 Roze E, Coelho-Braga MC, Gayraud D, et al. Head tremor in Parkinson's disease. Mov Disord 2006;21:1245-8.

36 Rajput AH, Gibb WR, Zhong $\mathrm{XH}$, et al. Dopa-responsive dystonia: pathological and biochemical observations in a case. Ann Neurol 1994;35:396-402.

37 Rajput AH, Rozdilsky B, Hornykiewicz 0, et al. Reversible drug-induced parkinsonism. Clinicopathologic study of two cases. Arch Neurol 1982;39:644-6.

38 Sudarsky L, Ronthal M. Gait disorders among elderly patients. A survey study of 50 patients. Arch Neurol 1983;40:740-3.

39 Weiner WJ, Nora LM, Glantz RH. Elderly inpatients: postural reflex impairment. Neurology 1984;34:945-7.

40 Poewe W, Scherfler C. Role of dopamine transporter imaging in investigation of parkinsonian syndromes in routine clinical practice. Mov Disord 2003;18(Suppl 7): S16-21.

41 Sawle GV, Leenders KL, Brooks DJ, et al. Dopa-responsive dystonia: [18F]dopa positron emission tomography. Ann Neurol 1991;30:24-30.

42 Snow BJ, Nygaard TG, Takahashi H, et al. Positron emission tomographic studies of dopa-responsive dystonia and early-onset idiopathic parkinsonism. Ann Neurol 1993;34:733-8. 